\title{
Do Bone Graft and Cracking of the Sclerotic Cavity Improve Fixation of Titanium and Hydroxyapatite-coated Revision Implants in an Animal Model?
}

\author{
Brian Elmengaard MD, PhD, Joergen Baas MD, PhD, Thomas Jakobsen MD, PhD, \\ Soren Kold MD, PhD, Thomas B. Jensen MD, PhD, Joan E. Bechtold PhD, \\ Kjeld Soballe MD, DSc
}

Published online: 23 August 2016

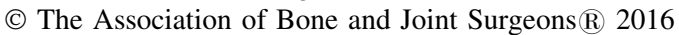

\begin{abstract}
Background We previously introduced a manual surgical technique that makes small perforations (cracks) through the sclerotic bone shell that typically forms during the process of aseptic loosening ("crack" revision technique). Perforating just the shell (without violating the proximal cortex) can maintain overall bone continuity while allowing marrow and vascular elements to access the implant surface. Because many revisions require bone graft to fill defects, we wanted to determine if bone graft could further increase implant fixation beyond what we have experimentally shown with the crack technique alone. Also,
\end{abstract}

One of the authors (JEB) received support from National Institutes of Health 42051.

All ICMJE Conflict of Interest Forms for authors and Clinical Orthopaedics and Related Research ${ }^{\mathbb{R}}$ editors and board members are on file with the publication and can be viewed on request.

Clinical Orthopaedics and Related Research ${ }^{\mathbb{R}}$ neither advocates nor endorses the use of any treatment, drug, or device. Readers are encouraged to always seek additional information, including FDAapproval status, of any drug or device prior to clinical use. Each author certifies that his or her institution approved the animal protocol for this investigation and that all investigations were conducted in conformity with ethical principles of research. The experimental work was performed at the Minneapolis Medical Research Foundation, Minneapolis, MN, USA. Specimen analysis was performed at Aarhus University, Aarhus, Denmark.

B. Elmengaard, J. Baas, S. Kold, J. E. Bechtold, K. Soballe Orthopaedic Research Laboratory, Department of Orthopaedic Surgery, Aarhus University Hospital, Aarhus, Denmark

T. Jakobsen

Department of Orthopaedic Surgery, Aalborg University

Hospital, Aalborg, Denmark

T. B. Jensen

Department of Plastic Surgery, Aarhus University Hospital,

Aarhus, Denmark because both titanium (Ti6Al4V) and hydroxyapatite (HA) implant surfaces are used in revisions, we also wanted to determine their relative effectiveness in this model.

Questions/purposes We hypothesized that both (1) allografted plasma-sprayed Ti6Al4V; and (2) allografted plasma-sprayed HA-coated implants inserted with a crack revision technique have better fixation compared with a noncrack revision technique in each case.

Methods Under approval from our Institutional Animal Care and Use Committee, a female canine animal model was used to evaluate the uncemented revision technique (crack, noncrack) using paired contralateral implants while implant surface (Ti6Al4V, HA) was qualitatively compared between the two (unpaired) series. All groups received bone allograft tightly packed around the implant. This revision model includes a cylindrical implant pistoning $500 \mu \mathrm{m}$ in a $0.75-\mathrm{mm}$ gap, with polyethylene particles, for 8 weeks. This engenders a bone and tissue response representative of the metaphyseal cancellous region of an aseptically loosened component. At 8 weeks, the original implants were revised and followed for an additional 4 weeks. Mechanical fixation was assessed by load, stiffness, and energy to failure when loaded in axial pushout. Histomorphometry was used to determine the amount and location of bone and fibrous tissue in the grafted gap.

\section{J. E. Bechtold}

Department of Orthopaedic Surgery, Minneapolis Medical Research Foundation, University of Minnesota, Minneapolis, MN, USA

J. E. Bechtold ( $\square)$

Orthopaedic Biomechanics Laboratory, Minneapolis Medical Research Foundation, Life Sciences Building, Suite 118, 700 South 10th Avenue, Minneapolis, MN 55415, USA e-mail: bechtold@umn.edu 
Results The grafted crack revision improved mechanical shear strength, stiffness, and energy to failure (for Ti6Al4V 27- to 69-fold increase and HA twofold increases). The histomorphometric analysis demonstrated primarily fibrous membrane ongrowth and in the gap for the allografted Ti6Al4V noncrack revisions. For allografted HA noncrack revisions, bone ongrowth at the implant surface was observed, but fibrous tissue also was present in the inner gap. Although both Ti6Al4V and HA surfaces showed improved fixation with grafted crack revision, and Ti6Al4V achieved the highest percent gain, HA demonstrated the strongest overall fixation.

Conclusions The results of this study suggest that novel osteoconductive or osteoinductive coatings and bone graft substitutes or tissue-engineered constructs may further improve bone-implant fixation with the crack revision technique but require evaluation in a rigorous model such as presented here.

Clinical Relevance This experimental study provides data on which to base clinical trials aimed to improve fixation of revision implants. Given the multifactorial nature of complex human revisions, such a protocoled clinical study is required to determine the clinical applicability of this approach.

\section{Introduction}

Revision arthroplasty is complex, combines structural and biologic solutions, and its durability is multifactorial $[5,6,13,28]$. One strategy to help increase longevity is through locally improving cancellous bone ingrowth in the acetabulum and metaphyseal regions of the hip and knee. In these regions, the surgeon may find a complete or segmental sclerotic bone shell that typically forms during the process of aseptic loosening. In a series of animal studies, we have tried to determine how the surgeon should approach the sclerotic bone shell to stimulate bone ingrowth and improve revision implant fixation. We introduced a surgical technique that cracks (perforates) through the sclerotic bone shell without damaging the cortex and that maintains the integrity of the long bone ("crack" revision technique) $[3,4,12]$. The goal for this technique was to disrupt the physical bone barrier (sclerotic shell) and to allow marrow and vascular elements to access the revision implant surface, thereby enhancing bone ingrowth. In a series of animal studies, we have shown that this crack revision technique improved fixation of nongrafted porous-coated titanium (Ti6Al4V) and plasmasprayed hydroxyapatite (HA) -coated revision implants compared with a "noncrack" revision technique where the bone shell is left in situ [8, 12]. The crack revision technique also improved the fixation of nongrafted plasma- sprayed HA-coated revision implants and increased new bone formation compared with the technique of reaming where the entire bone shell was removed [2]. We also have shown HA to improve fixation in a noncrack revision experimental animal model compared with Ti6Al4V $[19,23,25]$ and that allograft packed into a periimplant gap improves fixation in a variety of experimental and clinical settings [4, 7, 9, 22, 23, 27-29].

Although the previous animal studies have shown that the cracking revision technique may improve implant fixation compared with leaving the bone shell in situ or reaming, we also recognize that clinically, the human revision setting is different from the standardized experimental model. Clinically, the sclerotic bone shell may be irregular in shape and extension. If the bone shell is partially left intact, gaps may form between the bone bed and the revision implant. For this reason, and if cavitary bone defects exist as a result of the process of loosening, there may be a need for bone grafting to improve the stability of the revision implant. An experimental animal study in the revision setting showed that bone allograft markedly increases early stability of both plasma-sprayed Ti6Al4V and HA revision implants [23]. However, it is unknown whether there is a ceiling effect with either bone graft or the crack revision technique such that their combination may not further strengthen the implant interface beyond that achieved by each one alone. In our previous experimental animal studies we have not used bone allograft in conjunction with the cracking revision technique. The focus of this study is to investigate whether the cracking revision technique is also beneficial when the revision implants are impacted with bone allograft and whether there is a difference with implant surface.

We pose two hypotheses regarding implant fixation as determined by the primary endpoint: mechanical pushout (strength, stiffness, and energy to failure) and the secondary endpoint: histomorphometric indices of periimplant bone location and amount (bone volume, bone in contact with surface): Hypothesis 1: Allografted plasma-sprayed Ti6Al4V implants inserted with crack revision technique have better fixation compared with allografted plasmasprayed Ti6Al4V implants inserted with a noncrack revision technique. Hypothesis 2: Allografted plasma-sprayed HA-coated implants inserted with a crack revision technique have better fixation compared with allografted plasma-sprayed HA revision implants inserted with the noncrack revision technique. Although the studies cannot prove it, we postulate the mechanism of improvement with cracking would be the result of greater access of blood and marrow element to the bone surface, resulting in increased bone at the implant surface and in gap and less fibrous tissue. We postulate that the mechanism of improvement 
with HA surface would be the result of its osteoinductive properties, increasing bone at the implant surface.

\section{Materials and Methods}

Under approval from the Minneapolis Medical Research Foundation Institutional Animal Care and Use Committee (Protocol 01-17), we implemented our contralateral/paired controlled revision protocol $[3,4]$ in two separate groups of female canines (Table 1). One group received bilateral cylindrical Ti6Al4V implants in the distal femur (mean weight $23 \mathrm{~kg}$, range $20-27 \mathrm{~kg}$, mean age 15 months, $\mathrm{n}=8$ ). The other group received bilateral HA implants (mean weight $22 \mathrm{~kg}$, range 19-25 kg, mean age 10 months, $\mathrm{n}=$ 10). All animals were skeletally mature. All groups received bone allograft that was tightly packed in the periimplant space. All implants were uncemented.

We previously developed an experimental revision protocol in the canine knee that engenders a bone and tissue response representative of the metaphyseal region when surgically revising an aseptically loosened femoral component, described in more detail later [4, 26]. Although the protocol takes place in the knee, it is intended to represent a generic cancellous bone bed adjacent to the ingrowth portion of a joint replacement implant and with access to synovial fluid and loading. The revision protocol is briefly described here.

\section{Revision Protocol}

A micromotion device is inserted bilaterally in the animal's distal medial femoral condyle to replicate the motion of a loose prosthesis by providing a pistoning motion in a cancellous bone bed (Fig. 1). The cancellous bone bed is intended to represent a generic cancellous site adjacent to a joint replacement implant. The pistoning micromotion device anchor housing is inserted bilaterally as follows. Under general anesthesia and using a sterile surgical technique, a midline incision is made over each knee sequentially (both knees are operated on bilaterally in the same setting). The distal femur is exposed, the knee is flexed, and the weightbearing portion of the medial condyle is identified. A cannulated 7.5-mm step drill is inserted over a guidewire to produce a cavity with a $6 \mathrm{~mm} \times 1-\mathrm{cm}$ deep portion and an $8 \mathrm{~mm} \times 1-\mathrm{cm}$ superficial portion. The deep cavity is tapped using a tool that centralizes and stabilizes the tap. The anchor housing of the micromotion device is screwed into the deep hole using an instrument that stabilizes the screwdriver, thereby keeping the piston centralized within the cavity. The anchor housing includes an internal threaded piston designed to allow $500 \mu \mathrm{m}$ of motion on loading. Its internal spring extends the piston after loading. It is through this process that controlled

Series 1: Bilateral Ti Implant Surface $(n=8)$ Series 2: Bilateral HA Implant Surface $(n=9)$

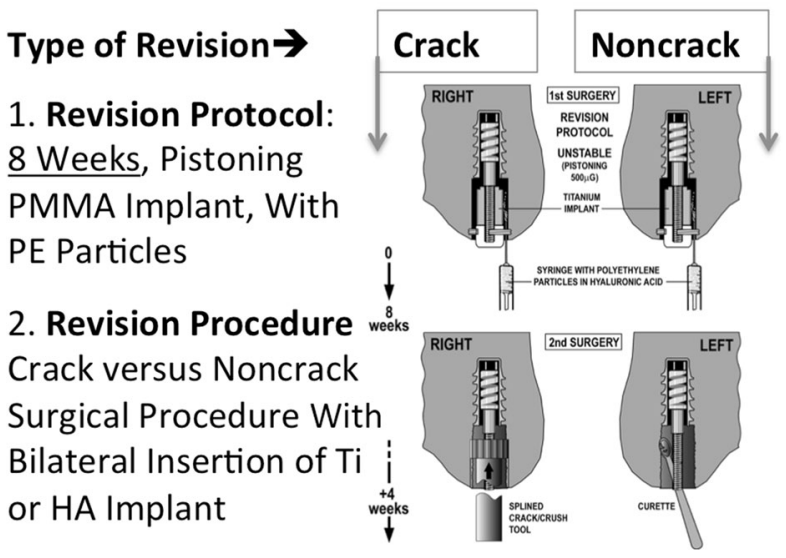

3. Outcome Measures

4 Weeks: Mechanical Pushout + Histomorphometry

Fig. 1 Two experimental animal series are shown with bilateral revision implantations in canine distal medial femoral condyles. The bone-implant interface represents cancellous metaphyseal or acetabular bone adjacent to ingrowth portion of the joint replacement implant. The 8-week revision protocol creates sclerotic bone shell and inflammatory tissue reaction. Implants are then revised bilaterally (Ti/ Series 1 or HA/Series 2) and followed for an 4 additional weeks. Components of the micromotion device are identified. $\mathrm{PE}=$ polyethylene.

Table 1. Two paired studies evaluating the crack revision technique as compared with the noncrack revision technique that retains the sclerotic bone shell*

\begin{tabular}{lll}
\hline Implant surface & Bone allograft & \\
\cline { 3 - 3 } & Ti6Al4V & HA \\
\hline Noncrack revision & $\mathrm{n}=8$ (left) & $\mathrm{n}=10$ (left) \\
Crack revision & $\mathrm{n}=8$ (right) & $\mathrm{n}=10$ (right) \\
\hline
\end{tabular}

* All implants receive impacted bone allograft; revision technique is evaluated pairwise for each implant surface (Ti or HA; Hypotheses 1 and 2); the combination of implant surface and revision technique is qualitatively evaluated as a result of the implants being unpaired (one implant from each series); HA = hydroxyapatite. 
Table 2. Results from the mechanical pushout test

\begin{tabular}{|c|c|c|c|c|c|c|c|}
\hline \multirow[t]{2}{*}{ Parameter } & \multicolumn{3}{|c|}{ Hydroxyapatite $(n=9)$} & \multirow[t]{2}{*}{$\mathrm{p}$ value } & \multicolumn{2}{|l|}{ Titanium $(\mathrm{n}=8)$} & \multirow[t]{2}{*}{$\mathrm{p}$ value } \\
\hline & $\begin{array}{l}\text { Crack revision } \\
\text { Mean (SD) }\end{array}$ & $\begin{array}{l}\text { Noncrack revision } \\
\text { Mean (SD) }\end{array}$ & $\begin{array}{l}\text { Mean difference } \\
(95 \% \mathrm{CI})\end{array}$ & & $\begin{array}{l}\text { Crack revision } \\
\text { Median (range) }\end{array}$ & $\begin{array}{l}\text { Noncrack revision } \\
\text { Median (range) }\end{array}$ & \\
\hline Shear strength (MPa) & $3.9(0.9)$ & $2.3(1.6)$ & $1.6(0.70-2.5)$ & 0.03 & $2.7(0.5-3.7)$ & $0.1(0-0.12)$ & 0.02 \\
\hline Energy to failure $\left(\mathrm{J} / \mathrm{m}^{2}\right)$ & $689(196)$ & $358(281)$ & $331(158-504)$ & 0.03 & $414(100-682)$ & $6(0-30)$ & 0.02 \\
\hline Stiffness $(\mathrm{MPa} / \mathrm{mm})$ & $21.8(7.6)$ & $10.5(7.9)$ & $11.3(5.8-16.8)$ & 0.01 & $10.7(0.8-14)$ & $0.3(0-1.3)$ & 0.01 \\
\hline
\end{tabular}

$\mathrm{CI}=$ confidence interval.

Table 3. Tissue at the bone-implant interface

\begin{tabular}{|c|c|c|c|c|c|c|c|}
\hline \multirow[t]{2}{*}{ Tissue $(\%)$} & \multicolumn{3}{|c|}{ Hydroxyapatite $(\mathrm{n}=9)$} & \multirow[t]{2}{*}{$\mathrm{p}$ value } & \multicolumn{2}{|l|}{ Titanium $(\mathrm{n}=8)$} & \multirow[t]{2}{*}{$\mathrm{p}$ value } \\
\hline & $\begin{array}{l}\text { Crack revision } \\
\text { Mean (SD) }\end{array}$ & $\begin{array}{l}\text { Noncrack revision } \\
\text { Mean (SD) }\end{array}$ & Mean difference $(95 \% \mathrm{CI})$ & & $\begin{array}{l}\text { Crack revision } \\
\text { Median (range) }\end{array}$ & $\begin{array}{l}\text { Noncrack revision } \\
\text { Median (range) }\end{array}$ & \\
\hline Bone & $51(6.3)$ & $38(16)$ & $13(4-22)$ & 0.02 & $12(4-23)$ & $0(0-0)$ & 0.01 \\
\hline Bone marrow & $36(19)$ & $17(19)$ & $19(5-33)$ & 0.02 & $81(23-87)$ & $0(0-0)$ & 0.02 \\
\hline Fibrous & $11(22)$ & $44(25)$ & $-33(-50$ to -16$)$ & 0.01 & $7(0-72)$ & $100(100-100)$ & 0.01 \\
\hline
\end{tabular}

$\mathrm{CI}=$ confidence interval.

motion occurs. After use of a cannulated tap, a $3 \mathrm{~mm} \times$ 8 -mm centralizing ring is screwed into the most superficial $3 \mathrm{~mm}$ of the superficial cavity. It ensures that the piston only moves axially and remains central in the gap. Polyethylene particles $(0.5-50 \mu \mathrm{m} ; 0.5 \times 108 ; 85 \%<12$ $\mu \mathrm{m})$ suspended in hyaluronic acid (Lifecore Biomedical, Chaska, MN, USA) represent wear debris and are injected into the superficial cavity. A $6 \mathrm{~mm} \times 1-\mathrm{cm}$ smooth polymethylmethacrylate (PMMA) implant is threaded onto the internal piston followed by a 5-mm polyethylene end plug. The end plug (1) protrudes past the articular cartilage (to cause implant loading during weightbearing); and (2) keeps the implant concentric within the gap. The soft tissues are closed in layers. By the first or second postoperative day, the animal is moving normally. Animals are socialized in groups and allowed full activity for the 8-week period, including a daily exercise period of 1 hour.

\section{Revision Procedure}

After 8 weeks of pistoning with polyethylene particles, a dense fibrous membrane develops in the original gap, and the bone at the original drill hole has coalesced into a sclerotic bone shell $[4,26]$. The volume of joint fluid and thickness of capsule are consistently observed to be increased (qualitative increase). At this time a second operation is performed. In a pairwise fashion, one of two surgical techniques is used to insert allografted revision implants into the revision cavity (Fig. 1).

\section{Noncrack Revision Procedure}

On the left side, meticulous removal of the fibrous membrane is performed. The sclerotic surface is scraped with a curette and the cavity cleaned and lavaged.

\section{Crack Revision Procedure}

On the right side, following the same fibrous membrane removal and lavage, a splined crack tool is introduced into the revision cavity. The crack tool is a cannulated cylinder of 8 $\mathrm{mm}$ diameter fitted with 12 sharp, axially orientated $0.2-\mathrm{mm}$ splines on the surface. It is advanced axially with hammer blows into the superficial cavity. This produces controlled cracking/perforation of the sclerotic shell and leaves disrupted bone pieces from the sclerotic shell. The cracking procedure produces multiple small $(<1 \mathrm{~mm})$ irregularly shaped pieces of bone that remain in place. Although clinically, insertion of a wedge-shaped femoral component can result in an intraoperative periprosthetic fracture in human joint replacement surgery, as a result of being surrounded by cancellous bone at the experimental implant site, the low profile of the splines on the custom tool $(0.2 \mathrm{~mm})$, and gentle insertion procedure, has not occurred in this model.

\section{Revision Implant}

After either procedure, the cavities are irrigated once with $10 \mathrm{cc}$ saline and gently suctioned so as not to disturb the 
Fig. 2A-D Histologic sections and corresponding $\mu \mathrm{CT}$ images ( $\mu \mathrm{CT}$ 40; Scanco Medical, Bassersdorf, Switzerland) were obtained from paired Ti6Al4V implants. The crack revision procedure is shown (A): bone (green) is seen filling the gap and bone ongrowth to the implant (black) is also observed. The corresponding $\mu \mathrm{CT}$ image (B) shows bone ingrowth and no signs of the sclerotic bone shell. The noncrack revision is shown (C); complete bone allograft resorption has occurred around the control implants. The implant is surrounded by a dense fibrous membrane (red). Corresponding $\mu \mathrm{CT}$ image (D) shows an intact and dense sclerotic bone shell, which has blocked bony integration of the implant. Bone graft inserted at the time of surgery has resorbed over the 4-week observation period.
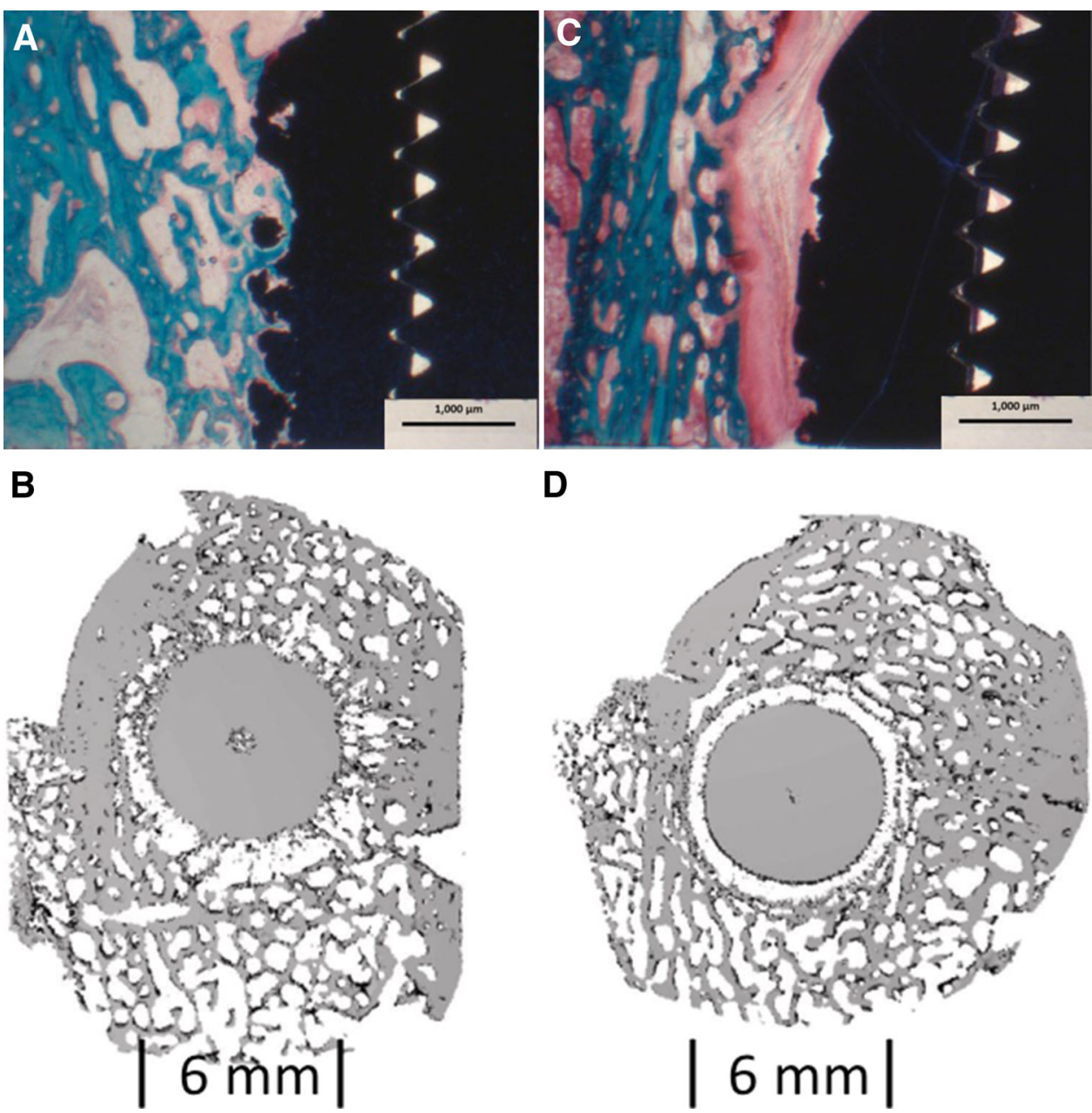

D

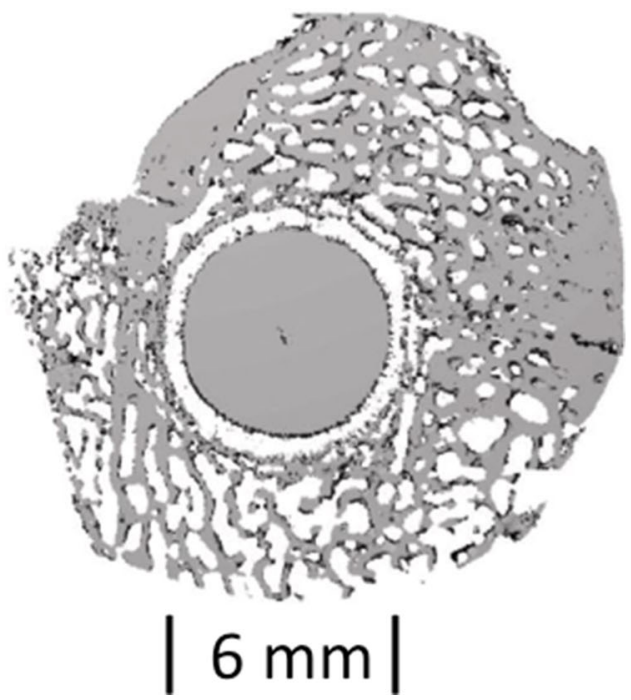

cracked pieces. After tapping, a larger centralizing ring is inserted into the superficial cavity.

A $6 \mathrm{~mm}$ diameter $\times 1-\mathrm{cm}$ long uncemented porous-coated (plasma-sprayed) HA or Ti6A14V cylindrical implant is advanced along the piston (coatings provided by Biomet, Inc, Warsaw, IN, USA). The roughened surface was created by plasma-spraying a coating of Ti6Al4V onto the Ti6Al4V core. This resulted in a pore size of $300 \mu \mathrm{m}$. A $50-\mu \mathrm{m}$ layer of $\mathrm{HA}(\mathrm{Ca} / \mathrm{P} 1.67)$ was plasma-sprayed onto the Ti6Al4V core. Roughness as measured by Ra was $47 \mu \mathrm{m}$ for Ti6Al4V implants and $41 \mu \mathrm{m}$ for HA implants [21]. After final seating on the piston, the deep portion of the implant contacts the anchor screw housing. As a result of an abutment on the revision implant, further pistoning motion is prevented. The revised implant is thereby intraarticular, stable, and loaded.

\section{Bone Allograft}

Batches of fresh-frozen allograft were prepared immediately before the surgical procedures. For each batch, long bones previously harvested under sterile conditions from dogs used in other research studies were wrapped in sterile saline-soaked gauze and stored frozen at $-80{ }^{\circ} \mathrm{C}$. Under sterile conditions, all cartilage and soft tissues were removed and the remaining cancellous bone was morselized using a standard surgical bone graft tool. Bone from different canine donors was mixed and portioned into 1$\mathrm{cm}^{3}$ aliquots, stored in sterile containers, and refrozen to $-20{ }^{\circ} \mathrm{C}$. During surgery, the bone allograft was inserted into the revision cavity using a specially modified curette and cylindrical impaction tool $[22,23]$. The procedure was repeated in approximately three to four steps until the entire revision cavity was filled with tightly packed allograft. A polyethylene end plug was inserted and the soft tissues were closed in layers. Animals were moving normally by the first or second postoperative day postrevision. For an additional 4 weeks, animals were again socialized in groups and were allowed full activity and a daily exercise period of 1 hour.

All animals were fully weightbearing within 48 hours of surgery. At the end of the 4-week revision observation 
Table 4. Tissue in the 0 - to $375-\mu \mathrm{m}$ gap for grafted hydroxyapatite implants $(n=19)$

\begin{tabular}{lllll}
\hline Tissue $(\%)$ & $\begin{array}{l}\text { Crack revision } \\
\text { Mean (SD) }\end{array}$ & $\begin{array}{l}\text { Noncrack revision } \\
\text { Mean (SD) }\end{array}$ & Mean difference (95\% CI) \\
\hline Bone & $35(6.3)$ & $20(16)$ & $15(6.4-24)$ & 0.03 \\
Bone marrow & $41(28)$ & $22(19)$ & $19(1.7-36)$ & 0.02 \\
Fibrous & $22(32)$ & $67(32)$ & $-45(-68$ to -22$)$ & 0.01 \\
\hline
\end{tabular}

$\mathrm{CI}=$ confidence interval.

Fig. 3A-B Histologic sections were obtained from paired HA implants. The crack revision procedure is shown $(\mathbf{A})$ : bone (green) is seen filling the gap and bone ongrowth to the implant (black) is also observed. The noncrack procedure is shown $(\mathbf{B})$ : complete bone allograft resorption has occurred around the control implants. Bone ongrowth is observed at the implant surface and in the gap fibrous tissue is present along with bone.
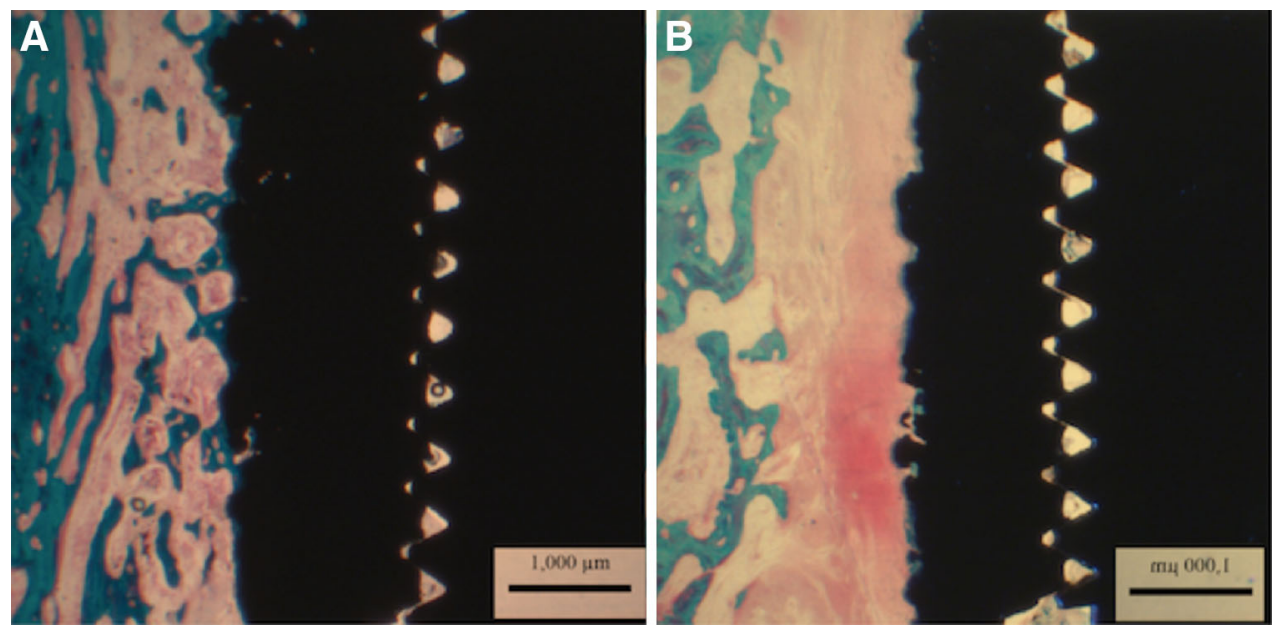

period, the animals were sedated, anesthetized, and euthanized according to the institutionally approved protocol. Distal femoral condyles were dissected free and frozen until specimen preparation.

\section{Specimen Preparation}

Bone blocks were thawed to room temperature. Alignment of the distal femur specimen through the implant axis ensured cuts perpendicular to the implant axis. At the site of the 1-cm cylindrical implant, the distal femur was cut transversely into two pieces. The outer 3-mm implant-bone block was stored for mechanical testing. The remaining 6$\mathrm{mm}$ implant-bone block was prepared for histomorphometric staining and sectioning.

\section{Mechanical Analysis}

Mechanical axial pushout tests $(5.0 \mathrm{~mm} / \mathrm{min})$ were performed on the 3-mm transverse fresh frozen implant/bone sections (MTS Mini Bionix 858, Eden Prairie, MN, USA) $[3,4]$. Ultimate shear strength, apparent shear stiffness, and energy absorption to failure were derived from force-displacement curves using nominal data from individual implant diameters and specimen thicknesses. Strength, stiffness, and energy to failure represent different characteristics of mechanical fixation of the bone-implant interface and the ability of the implant to remain in place without becoming dislodged.

\section{Histomorphometric Analysis}

Histomorphometric specimens were dehydrated in graded ethanol (70\%-100\% containing $0.4 \%$ basic fuchsin), embedded in methylmethacrylate, and during sectioning counterstained using $2 \%$ light green [10]. To obtain unbiased volume estimates of the tissue in the gap, the implant was cut with the standard stereological method using vertical sections through the implant cylinder (instead of horizontal sections) [16]. Before sectioning and to maintain conditions consistent with unbiased measures, the embedded specimens with the cylindrical implant in situ were randomly rotated around the cylinder's vertical axis and four serial sections of 15 to $20 \mu \mathrm{m}$ were obtained from the central portion of the implant using a microtome (KDG-95; 151 MeProTech, Heerhugowaard, Holland). Histomorphometry was performed in a blinded manner using a stereologic image analysis system (CAST-Grid, Olympus, Denmark) $[8,12]$. Tissue ongrowth was defined as tissue in direct contact with the implant surface and was determined using a line-intercept technique. Tissue volume 
Table 5. Tissue in the 0 - to $750-\mu \mathrm{m}$ gap for grafted titanium implants $(\mathrm{n}=8)$

\begin{tabular}{lclr}
\hline Tissue $(\%)$ & $\begin{array}{l}\text { Crack revision } \\
\text { Median (range) }\end{array}$ & $\begin{array}{l}\text { Noncrack revision } \\
\text { Median (range) }\end{array}$ & $\mathrm{p}$ value \\
\hline Bone & $28(14-36)$ & $13(7-19)$ & 0.02 \\
Bone marrow & $69(51-78)$ & $19(8-24)$ & 0.02 \\
Fibrous & $1(0-22)$ & $70(46-85)$ & 0.02 \\
\hline
\end{tabular}

percentages were estimated in the allografted gap adjacent to the implant by a point-counting technique.

Number of Implants, Statistical Methods, and Power

Eight animals received bilateral Ti6Al4V implants (eight animals, 16 implants) and 10 animals received bilateral HA implants (10 animals, 20 implants). Between the conduct of the two series, we increased the number of specimens per group to avoid loss of significance if inadvertent specimen loss occurred or if nonparametric data occurred. In the Ti6Al4V series, no animals were lost $(\mathrm{n}=8)$, and in the HA series, one animal was lost as a result of clinical signs of infection at the time of revision surgery $(n=9)$. The sample size calculation was based on histomorphometric and mechanical data obtained from earlier studies using the same experimental model. The SD was set to $50 \%$, minimal relevant difference was set to $50 \%$, risk of type I error $(\alpha)$ was 0.05 , and risk of type II error $(\beta)$ was 0.20 . With these assumptions a minimum of eight animals had to be included in each study. STATA Intercooled 8.0 statistical software (STATA, College Station, TX, USA) was used to test differences between pairs. For Ti6Al4V implants, the Wilcoxon signed-rank test was applied, because differences between the paired values were not normally distributed. Results are given as median and interquartile range. For HA implants, data followed a normal distribution, and Student's paired t-test was applied for comparison of groups. Within Ti6Al4V and HA implant groups, significance was considered for two-tailed $\mathrm{p}<0.05$.

\section{Results}

For Ti6Al4V revision implants, impacted bone allograft in combination with the crack revision technique resulted in increased mechanical fixation (shear strength, energy to failure, and stiffness by axial pushout test) compared with allografting with a noncrack revision technique in which the sclerotic bone shell was left in situ (Table 2). No bone allograft remained in the gap for either revision technique (bone was identified only as woven and non-Haversian). The crack revision technique resulted in increases of bone and bone marrow at the implant surface (ongrowth) (Table 3; Fig. 2) and $97 \%$ of the grafted gap surrounding the revision implant consisted of bone or bone marrow (Table 4; median and interquartile range; bone $28 \%$ [18\%$31 \%$ ] and bone marrow 69\% [54\%-73\%]). The noncrack revision technique resulted in $100 \%$ fibrous tissue ongrowth and the allografted gap was dominated by fibrous tissue (70\% [47\%-81\%]) (Table 4).

For plasma-sprayed HA-coated revision implants, impacted bone allograft in combination with the crack revision technique resulted in increased mechanical fixation (measured by an axial pushout test) compared with allografting with a noncrack revision technique (Table 2). The crack revision resulted in increased bone and bone marrow ongrowth (Table 3; Fig. 3). In the inner half of the grafted gap, $89 \%$ of the tissue was bone and bone marrow with the crack revision technique versus only $56 \%$ with the noncrack revision technique (Table 5; mean and SD).

Although a direct statistical comparison was not performed between the series with allografted plasma-sprayed Ti6Al4V revision implants and the series with allografted plasma-sprayed HA-coated revision implants, the data suggest that HA-coated implants obtain higher mechanical fixation than Ti6Al4V revision implants regardless of surgical technique (Table 2). For allografted HA revision implants, a twofold increase in stiffness and less than twofold increases in shear strength and total energy absorption were seen in favor of the crack revision technique. For allografted Ti6Al4V revision implants, the relative increase in mechanical fixation was higher because we observed a 27 -fold increase in shear strength, a 69 -fold increase in total energy absorption, and a 35-fold increase in stiffness in favor of the crack revision technique.

\section{Discussion}

Primary joint replacements have good outcomes and longevity, but with longer life spans and patients receiving joint replacements at a younger age, there is an increasing pool of joints that loosen and need to be revised $[5,6]$. These revisions are more complex operative procedures, often presenting with bone loss and a full or partial sclerotic bone shell surrounding the implant $[26,28]$. In our 
two-stage experimental canine revision joint replacement model, we have previously shown the benefit of a "crack" procedure to locally make small perforations in the sclerotic shell and thus allow marrow and blood vessels to reach the implant surface $[8,12]$. We also have shown the consistent benefit of bone graft $[4,7]$. What we did not know is whether there is a ceiling effect for the crack revision procedure, ie, whether added benefit would occur when using crack revision and bone graft together; we found that there was not a ceiling effect. Both Ti6Al4V and HA implants received benefit by bone grafting a crack revision. Although not a statistical comparison of paired specimens, we noted that although grafted Ti6Al4V implants received a larger relative benefit from the crack revision procedure, HA implants did achieve the highest actual fixation strength.

Limitations in this study include those inherent to the controlled animal model representing revision of a human implant. The model represents a cancellous environment such as the proximal metaphyseal region of a stemmed component but does not attempt to recreate the complexity of a human revision setting and bone bed (bone loss, medialization or shortening, and loss of structural competence of remaining bone) $[15,20,24]$. The revision protocol in this animal model uses a bilateral loaded implant, pistoning in the presence of polyethylene particles, and presents at revision with features characterizing loosened human implants [3, 4]. Although the protocol does not include structural aspects related to bone loss such as a patulous canal at revision surgery, the level of experimental control on conditions at the local bone-implant interface and the ability to conduct within-animal comparisons importantly improve reproducibility. Canines are used, because their cancellous bone represents the structure and density of human bone [17], but they do have more rapid bone turnover than humans and the animals in this study were young (albeit skeletally mature) rather than the typical revision joint recipient ( $>70$ years). However, like seen clinically, we have shown experimentally that the noncrack revision produces worse fixation than a primary implant. This is presumably because it has not undergone the deleterious bone and tissue changes that occur during the two-stage revision protocol $[4,23]$. Only one observation period (4 weeks) was evaluated, which represents the early reaction to the implant. We justify this because in humans, early excessive migration and poor fixation have been shown to be associated with later loosening $[11,14,18]$. Furthermore, in this model we have shown a 4-week observation period to reliably distinguish among treatment efficacy $[21,23]$. Two different batches of allograft were used for the Ti6Al4V and HA series. Furthermore, only fresh-frozen allograft, without irradiating, washing, or other processing, was used. It is not known if results would be the same with processed allograft or if batch-to-batch differences exist. Only one sex (female) was investigated, and it is not known whether results would be different with male animals.

We found that the crack revision technique with allografted Ti6Al4V implants improved fixation compared with the noncrack revision. This was shown both by mechanical measures as well as by histomorphologic presence of increased periimplant bone. An allografted Ti6Al4V revision implant in an intact sclerotic bone shell resulted in pure fibrous fixation at the implant interface (ongrowth) and fibrous tissue in the majority of the original gap. Fibrous fixation predictably resulted in inferior mechanical properties. When the sclerotic bone shell was cracked, bone and bone marrow covered the majority of the implant interface and the gap. This change in tissue distribution improved the mechanical fixation. Hence, an intact sclerotic bone shell seems to create an unfavorable environment in which allograft is of little benefit because it is resorbed and replaced by fibrous tissue. The increased mechanical and histomorphometric indices of bone-implant fixation with cracking of the sclerotic shell in the presence of bone allograft may also be related to the initial and later events in allograft incorporation. Initial inflammation, revascularization, and osteoinduction occur rapidly. The formation of bone requires a specialized cell and signaling response. In this coordinated response, the presence of osteotrophic growth factors, RGD (Arg-GlyAsp) containing extracellular matrix proteins, oxygen, and nutrients encourages cells to adhere and differentiate [1]. Later events in the incorporation and mineralization of bone allograft may also be aided by the sclerotic shellcracking procedure. After the initial response, mesenchymal cells of the host are recruited to differentiate into osteoblasts, and new bone grows on preexisting dead trabeculae. Cracking the sclerotic shell may facilitate the recruitment of these mesenchymal cells, both from the marrow space and through improved vascular access to the interface [26]. Subsequently, entrapped necrotic allografted bone is partially resorbed by osteoclasts, and in time, given conductive conditions, the allograft is replaced by osteoid and finally mineralized bone. The intact sections of the remaining sclerotic shell can also function as a scaffold fostering this bone (and vessel) growth.

We found that the crack revision technique with allografted HA implants also improved fixation compared with the noncrack revision. This was shown for both by mechanical measures as well as by histomorphometric presence of increased periimplant bone. The same biologic benefits of the cracking technique, as described previously for Ti6Al4V implants, are presumed to be relevant also to HA implants. Of importance, a ceiling effect where allografted HA implants would receive no 
further benefit of the cracking revision procedure was not demonstrated.

We found that allografted $\mathrm{Ti}$ implants received more relative percentage gain from the crack revision technique than HA implants, but HA implants qualitatively demonstrated greater overall fixation compared with Ti6Al4V. This is supported both by the mechanical measures as well as the histomorphometric presence of increased periimplant bone. This relative greater increase in mechanical fixation for Ti6Al4V revision implants was associated with relatively larger increases in bone and bone marrow with the crack revision technique. With either an allografted $\mathrm{Ti6A14V}$ or HA-coated revision implant, we also observed increased mechanical fixation when the sclerotic bone shell was cracked. However, the tissue distribution around the HA-coated revision was different compared with Ti6Al4V revision implants. When the sclerotic bone shell was intact (noncrack revision), HA implants demonstrated bone ongrowth at the surface. Despite this bone ongrowth with HA, both Ti6Al4V and HA allografted implants without cracking demonstrated a similar amount of fibrous tissue in the original gap. When the bone shell was cracked, reduced fibrous tissue was present in the gap. The phenomenon of a relatively high percentage of bone on a HA-coated surface and less bone in the gap has been observed in prior studies [21]. It is likely the result of HA's ability to adhere extracellular proteins, which stimulate bone growth to the surface [1]. Like in earlier studies [8, 12], the primary effect of the cracking procedure is reduction in fibrous capsule formation both at the interface and in the surrounding gap. Fibrous capsule formation is of great concern in hip revision arthroplasty because large quantities of fibrous tissue may compromise both the shortand long-term stability of the implants.

In summary, this study shows that the technique of cracking or locally disrupting the sclerotic bone shell results in increased fixation for both Ti6Al4V- and HAcoated allografted revision implants with a greater relative percentage increase for Ti6Al4V but greatest absolute fixation for HA. The absence of bone allograft after 4 weeks indicates a high level of cellular turnover in the revision setting. The presence of allograft had no positive effect on the fixation when used with Ti6Al4V revision implants when the sclerotic shell was left in situ (noncrack), whereas with HA implants, allograft was associated with bone ongrowth whether or not an intact sclerotic shell were present (crack and noncrack). Of concern, however, for HA-coated implants, high amounts of fibrous tissue were also seen, which potentially could eventually compromise long-term fixation. This in vivo canine animal study evaluating allograft further supports that the simple technique of cracking the sclerotic shell may be an addition to the surgeon's armamentarium for improving revision fixation in metaphyseal bone and acetabular sockets. The technique is bone-sparing while at the same time providing access to the vascular compartments of the bone and can be accomplished with a standard small osteotome, although purpose-designed instruments could also be considered. These findings suggest the importance of implant surface bioactivity as well as the role of bone graft in improving bone-implant fixation. Novel osteoconductive or osteoinductive coatings and bone graft substitutes or tissueengineered constructs may further improve bone-implant fixation with the crack revision technique but require evaluation in a rigorous model such as presented here. Although these experimental implants are clinically based, the results of this study should be interpreted with the constraints of this particular experimental loaded articular gap implant model and revision protocol.

Acknowledgments We thank Jane Pauli, Xinqian Chen, and Olivier Mouzin for their excellent technical assistance. Biomet, Inc (Warsaw, IN, USA) donated the PMMA implants and Ti6Al4V and HA implant coatings.

\section{References}

1. Anselme K. Osteoblast adhesion on biomaterials. Biomaterials. 2000;21:667-681.

2. Baas J, Elmengaard B, Jakobsen T, Bechtold J, Soballe K. Crack revision improves fixation of uncemented HA-coated implants compared with reaming: an experiment in dogs. Clin Orthop Relat Res. 2009;467:2638-2643.

3. Bechtold JE, Kubic V, Søballe K. A controlled experimental model of revision implants: Part I. Development. Acta Orthop Scand. 2001;72:642-649.

4. Bechtold JE, Mouzin O, Kidder L, Søballe K. A controlled experimental model of revision implants: Part II. Implementation with loaded titanium implants and bone graft. Acta Orthop Scand. 2001;72:650-656.

5. Bozic KJ, Kurtz SM, Lau E, Ong K, Vail TP, Berry DJ. The epidemiology of revision total hip arthroplasty in the United States. J Bone Joint Surg Am. 2009;91:128-133.

6. Bozic KJ, Lau E, Ong K, Chan V, Kurtz S, Vail TP, Rubash HE, Berry DJ. Risk factors for early revision after primary total hip arthroplasty in Medicare patients. Clin Orthop Relat Res. 2014;472:449-454.

7. Daugaard H, Elmengaard B, Andreassen TT, Baas J, Bechtold JE, Soballe K. The combined effect of parathyroid hormone and bone graft on implant fixation. J Bone Joint Surg Br. 2011;93:131-139.

8. Elmengaard B, Bechtold JE, Chen X, Søballe K. Fixation of hydroxyapatite-coated revision implants is improved by the surgical technique of cracking the sclerotic bone rim. J Orthop Res. 2009;27:996-1001.

9. Gie GA, Linder L, Ling RS, Simon JP, Slooff TJ, Timperley AJ. Impacted cancellous allografts and cement for revision total hip arthroplasty. J Bone Joint Surg Br. 1993;75:14-21.

10. Gotfredsen K, Budtz-Jörgensen E, Jensen LN. A method for preparing and staining histological sections containing titanium implants for light microscopy. Stain Technol. 1989;64:121-127.

11. Klerken T, Mohaddes M, Nemes S, Kärrholm J. High early migration of the revised acetabular component is a predictor of 
late cup loosening: 312 cup revisions followed with radiostereometric analysis for 2-20 years. Hip Int. 2015;25:471-476.

12. Kold S, Bechtold JE, Mouzin O, Elmengaard B, Chen X, Soballe K. Fixation of revision implants is improved by a surgical technique to crack the sclerotic bone rim. Clin Orthop Relat Res. 2005;432:160-166.

13. Lovald ST, Ong KL, Lau EC, Schmier JK, Bozic KJ, Kurtz SM. Mortality, cost, and downstream disease of total hip arthroplasty patients in the medicare population. J Arthroplasty. 2014;29:242246.

14. Nieuwenhuijse MJ, Valstar ER, Kaptein BL, Nelissen RG. Good diagnostic performance of early migration as a predictor of late aseptic loosening of acetabular cups: results from ten years of follow-up with roentgen stereophotogrammetric analysis (RSA). J Bone Joint Surg Am. 2012;94:874-880.

15. Ong KL, Lau E, Suggs J, Kurtz SM, Manley MT. Risk of subsequent revision after primary and revision total joint arthroplasty. Clin Orthop Relat Res. 2010;468:3070-3076.

16. Overgaard S, Søballe K, Jørgen H, Gundersen G. Efficiency of systematic sampling in histomorphometric bone research illustrated by hydroxyapatite-coated implants: optimizing the stereological vertical-section design. J Orthop Res. 2000;18:313321.

17. Pearce AI, Richards RG, Milz S, Schneider E, Pearce SG. Animal models for implant biomaterial research in bone: a review. Eur Cell Mater. 2007;13:1-10.

18. Pijls BG, Nieuwenhuijse MJ, Fiocco M, Plevier JW, Middeldorp $\mathrm{S}$, Nelissen RG, Valstar ER. Early proximal migration of cups is associated with late revision in THA: a systematic review and meta-analysis of 26 RSA studies and 49 survival studies. Acta Orthop. 2012;83:583-591.

19. Sandiford NA, Skinner JA, East DJ, Butler-Manuel A, Hinves BL, Shepperd JA. Primary total hip arthroplasty with hydroxyapatite coated titanium femoral stems. Does design philosophy influence long term outcome? Results of a prospective randomised controlled trial with follow-up of 10-15 years. Acta Orthop Belg. 2014;80:372-379.
20. Smith MA, Deakin AH, Allen D, Baines J. Midterm outcomes of revision total hip arthroplasty using a modular revision hip system. J Arthroplasty. 2016;31:446-450.

21. Søballe K. Hydroxyapatite ceramic coating for bone implant fixation. Acta Orthop Scand. 1993;64:1-58.

22. Søballe K, Jensen TB, Mouzin O, Kidder L, Bechtold JE, Differential effect of a bone morphogenetic protein-7 (OP-1) on primary and revision loaded, stable implants with allograft. $J$ Biomed Mater Res A. 2004;71:569-576.

23. Søballe K, Mouzin ORG, Kidder LA, Overgaard S, Bechtold JE. The effects of hydroxyapatite coating and bone allograft on fixation of loaded experimental primary and revision implants. Acta Orthop Scand. 2003;74:239-247.

24. Solomon LB, Costi K, Kosuge D, Cordier T, McGee MA, Howie DW. Revision total hip arthroplasty using cemented collarless double-taper femoral components at a mean follow-up of 13 years (8 to 20): an update. Bone Joint J. 2015;97:1038-1045.

25. Syed MA, Hutt NJ, Shah N, Edge AJ. Hydroxyapatite ceramic-coated femoral components in young patients followed up for 17 to 25 years: an update of a previous report. Bone Joint J. 2015;97:749-754.

26. Vestermark MT, Bechtold JE, Swider P, Søballe K. Mechanical interface conditions affect morphology and cellular activity of sclerotic bone rims forming around experimental loaded implants. J Orthop Res. 2004;22:647-652.

27. Warth LC, Callaghan JJ, Liu SS, Klaassen AL, Goetz DD, Johnston RC. Thirty-five-year results after Charnley total hip arthroplasty in patients less than fifty years old. A concise follow-up of previous reports. J Bone Joint Surg Am. 2014;96:1814-1819.

28. Wimmer MD, Randau TM, Deml MC, Ascherl R, Nöth U, Forst R, Gravius N, Wirtz D, Gravius S. Impaction grafting in the femur in cementless modular revision total hip arthroplasty: a descriptive outcome analysis of 243 cases with the MRP-TITAN revision implant. BMC Musculoskelet Disord. 2013;14:19.

29. Wraighte PJ, Howard PW. Femoral impaction bone allografting with an Exeter cemented collarless, polished, tapered stem in revision hip replacement: a mean follow-up of 10.5 years. J Bone Joint Surg Br. 2008;90:1000-1004. 